ARTICLE

\title{
Hot carriers perspective on the nature of traps in perovskites
}

\author{
Marcello Righetto (1) 1,3, Swee Sien Lim¹,3, David Giovanni (iD) 1,3, Jia Wei Melvin Lim (i) 1,2, Qiannan Zhang (i) 1, \\ Sankaran Ramesh (i) ${ }^{1,2}$, Yong Kang Eugene Tay (i) ${ }^{1} \&$ Tze Chien Sum (ib) ${ }^{1 凶}$
}

Amongst the many spectacular properties of hybrid lead halide perovskites, their defect tolerance is regarded as the key enabler for a spectrum of high-performance optoelectronic devices that propel perovskites to prominence. However, the plateauing efficiency enhancement of perovskite devices calls into question the extent of this defect tolerance in perovskite systems; an opportunity for perovskite nanocrystals to fill. Through optical spectroscopy and phenomenological modeling based on the Marcus theory of charge transfer, we uncover the detrimental effect of hot carriers trapping in methylammonium lead iodide and bromide nanocrystals. Higher excess energies induce faster carrier trapping rates, ascribed to interactions with shallow traps and ligands, turning these into potent defects. Passivating these traps with the introduction of phosphine oxide ligands can help mitigate hot carrier trapping. Importantly, our findings extend beyond photovoltaics and are relevant for low threshold lasers, light-emitting devices and multi-exciton generation devices.

\footnotetext{
${ }^{1}$ Division of Physics and Applied Physics, School of Physical and Mathematical Sciences, Nanyang Technological University, 21 Nanyang Link, Singapore 637371 Singapore. ${ }^{2}$ Energy Research Institute @NTU (ERI@N), Interdisciplinary Graduate School, Nanyang Technological University, 50 Nanyang Avenue, Singapore 639798, Singapore. ${ }^{3}$ These authors contributed equally: Marcello Righetto, Swee Sien Lim, David Giovanni. ${ }^{凶}$ email: Tzechien@ntu.edu.sg
} 
ybrid lead halide perovskites (LHPs) are thrust into the limelight of semiconductor research by the advances they bring into the field of solution-processed optoelectronics ${ }^{1}$ spanning photovoltaics ${ }^{2}$, light-emitting devices ${ }^{3}$, lasers ${ }^{4}$, and spintronics ${ }^{5}$. Surprisingly, LHP-based devices can achieve extraordinary performances despite possessing large densities of point defects. These defects arise from solution-processing (about $10^{16}$ to $10^{17} \mathrm{~cm}^{-3}$ for thin films) and consist predominantly of shallow traps with a smaller population of deep trap sites ${ }^{6-8}$. The latter act as non-radiative Shockley-Read-Hall recombination centers and are detrimental for device operations ${ }^{9,10}$. Past defect studies focused almost exclusively on deep trap states (such as lead vacancies, $\mathrm{V}_{\mathrm{Pb}}$ ), trying to mitigate their detrimental role in LHP by formulating design and synthetic rules ${ }^{11-13}$. In contrast, carriers trapped in shallow defect sites have a high probability of detrapping and are thus believed not to contribute significantly to non-radiative recombination rates $^{8}$. Because these shallow traps do not affect the electronic and optical properties of LHPs, this class of materials is commonly termed 'defect-tolerant' ${ }^{14,15}$.

After several years of relentless growth, efficiency enhancements in perovskite-based devices are reaching a plateau. This calls for a more in-depth study of defects in LHPs, a reconsideration of the role of shallow traps and their bearing on defect tolerance $^{13}$. Many of these applications rely on high-energy, nonresonant excitations, e.g., GaN back-illumination or high-energy charge injection (around $1 \mathrm{eV}$ excess energy above the band-gap) in light-emitting devices, and violet/blue sunlight conversion in solar cells. Hence, for them to achieve high performances, the defect tolerance of LHPs should also extend to high-energy excitations. The study of excitation energy-resolved photoluminescence quantum yield (PLQY) provides an insight into how incident energy affects the interaction between photogenerated carriers and traps. For instance, in molecular systems, the fast internal conversion process (i.e., cooling) and the absence of traps make the PLQY unsusceptible to the excitation energy ${ }^{16,17}$. It is essential to note that excitation energydependent PLQYs have a direct impact on device performance, and they can occur in all condensed matter systems due to the coupling between hot carriers and traps. Several recent works ${ }^{18,19}$ reported conflicting results on the presence of this coupling, thereby leaving an open question.

Perovskite nanocrystals (PNCs) provide an exciting platform to investigate the extent of defect tolerance to higher energy states, owing to (i) their higher PLQY that facilitate the use of fluorescence techniques, (ii) the introduction of surfaces as inherently defective sites, and (iii) the possibility of controlling the passivation of these defects with effective surface chemistry tools ${ }^{20-22}$. In recent years, PNCs took the nanocrystals field by storm, outpacing traditional II-VI semiconductor NCs in many of their principal applications, e.g., lower MEG and ASE thresholds, longer-lived hot carriers and larger multiphoton cross-sections ${ }^{23-26}$. PNCs have narrow linewidths $(12-30 \mathrm{~nm})$, an ultra-wide color gamut $(+140 \%$ of NTSC standard), and PLQYs reaching $90 \%$ and higher for bare core $\mathrm{NCs}^{27,28}$. The possibility of retaining high PLQY without extensive engineering of their surfaces, is the hallmark of a defecttolerant electronic structure 27,29 .

Herein, we demonstrate that $\mathrm{MAPbX}_{3}(\mathrm{X}=\mathrm{I}, \mathrm{Br})$ PNCs exhibit a substantial excitation energy-dependence on the PLQY, thus setting a fundamental limit to their photon conversion efficiencies. Using pump-probe (PP) and pump-push-probe (PPP) ultrafast spectroscopy measurements supported with phenomenological modeling, we demonstrate how hot carrier trapping mechanisms cause these excitation energy-dependent carrier losses in PNCs. In addition, we prove that it is possible to reduce their traps' electronic coupling with hot carriers by performing a post-synthetic ligand exchange to passivate some of these traps.
By uncovering these mechanisms, we aim to provide deeper insights into the photophysics of defect-tolerant PNCs and show that this tolerance may not apply to hot carriers. Importantly, these findings exemplify the need for further efforts in developing synthetical approaches, such as post-synthetic treatments, alternative types of ligands, and core-shell heterostructures that can decouple and mitigate the deleterious effects of defects on the hot carriers in these systems.

\section{Results}

Excitation energy-dependent PLQY in PNCs. We synthesized ambient-condition stable colloidal suspensions of $\mathrm{MAPbX}_{3}(\mathrm{X}=\mathrm{I}$, $\mathrm{Br}$ ) nanocrystals by a modification of the previously reported ligand

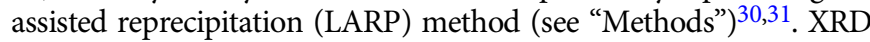
demonstrates the formation of a cubic structure (space group $P m \overline{3} m$ ), and TEM micrographs (Supplementary Figs. 1-3) confirm the quality of the synthesized samples: the particle size distribution obtained by statistical analysis is analogous to previous reports and between the two samples: $\mathrm{MAPbBr}_{3}$ and $\mathrm{MAPbI}_{3} \mathrm{NCs}$, with average radii of $4.2 \pm 1.3 \mathrm{~nm}$ and $5.5 \pm 1.5 \mathrm{~nm}$, respectively. For these two samples, we studied the role of excess photoexcitation energy above the band-gap (henceforth referred to as $\delta_{\mathrm{E}}$ ), in determining the PLQY of the PNCs. The effect of $\delta_{\mathrm{E}}$ in the PL of molecular and inorganic solids reflects the efficiency of the internal conversion or intraband relaxation. The PLQY spectra plotted as a function of $\delta_{\mathrm{E}}$, also known as 'photo-action' spectra, therefore contain information on hot carrier effects: dynamic processes occurring during the relaxation of high-energy carriers to the band edge ${ }^{32}$. These measurements were done with low-excitation intensities, as much as the techniques allow, to keep our focus on trapping and carrier loss mechanisms in the PNCs ${ }^{4}$.

Notably, the photo-action spectra of $\mathrm{MAPbBr}_{3}$ and $\mathrm{MAPbI}_{3}$ nanocrystals in Fig. 1a, b show substantial carrier losses at higher excitation energies. In $\mathrm{MAPbI}_{3} \mathrm{NCs}$, the PLQY drops from $25 \%$ when $\delta_{\mathrm{E}}=0.35 \mathrm{eV}$ to $18 \%$ when $\delta_{\mathrm{E}}=2.05 \mathrm{eV}$, thereby leading to a loss of approximately $30 \%$ of the carrier conversion when highenergy excitations are employed. For $\mathrm{MAPbBr}_{3} \mathrm{NCs}$, the PLQY plummets from 76 to $53 \%$ when $\delta_{\mathrm{E}}$ increases from $0.45 \mathrm{eV}$ to $1.25 \mathrm{eV}$. As reported in Supplementary Fig. 4, similar results were obtained by measuring the photoluminescence excitation spectrum and taking its ratio to the absorption spectrum. The pronounced losses even in the high-PLQY $\mathrm{MAPbBr}_{3} \mathrm{NCs}$ suggest that these effects are not correlated with the absolute value of the PLQY. Rather, these observations suggest that highly energetic excitations experience enhanced interactions with the trap sites, as compared with relaxed carriers. The implications of excitation energy-dependent PLQY in LHP nanostructures are two-fold: (i) imposing reconsiderations over the defect tolerance of LHPs; (ii) drawing attention to applications and devices that rely on the broadband absorber nature of PNCs. Indeed, potential applications of $\mathrm{MAPbI}_{3} \mathrm{NCs}$ in light-emission, lasing, and photovoltaics, as illustrated in Fig. 1a, rely on high-energy excitation and therefore are expected to experience lower conversion efficiencies.

Although the photophysics of $\mathrm{MAPbI}_{3}$ and $\mathrm{MAPbBr}_{3} \mathrm{NCs}$ exhibit differences, the trends in the photo-action spectra are comparable: sharp decreases in PLQY (zone I) that ease off at higher $\delta_{\mathrm{E}}$ (zone II). Figure 1c shows the absorption and photoluminescence (PL) spectrum of $\mathrm{MAPbI}_{3}$ and $\mathrm{MAPbBr}_{3} \mathrm{NCs}$, which fall in different ranges of the visible spectrum. Using the Elliott formula, we deconvolved the contributions from free carrier absorption, Rayleigh scattering, and excitonic absorption to the absorption spectra of PNCs, as shown in Fig. $1 c^{33,34}$. A prominent excitonic feature in the $\mathrm{MAPbBr}_{3} \mathrm{NCs}$ spectrum can be observed in Fig. 1c, d, and from its higher exciton binding 

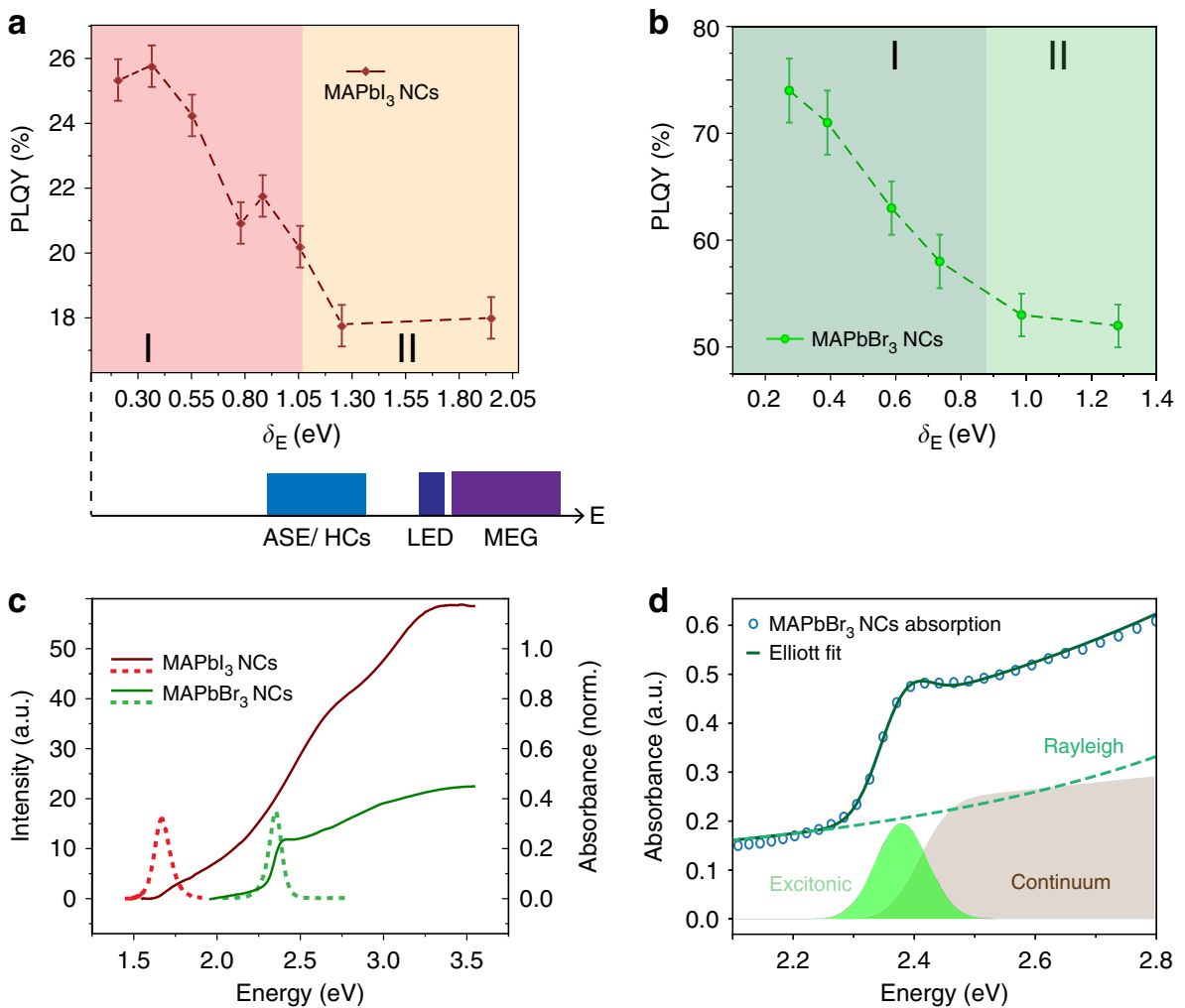

Fig. 1 Steady-state signatures of hot carrier losses. Excitation energy-dependent photoluminescence quantum yield spectra for (a) $\mathrm{MAPb}_{3}$ and (b) $\mathrm{MAPbBr}_{3} \mathrm{NCs}$, reported as a function of excess excitation energy $\left(\delta_{\mathrm{E}}\right)$ with respect to the bandgap obtained from the fitting the absorption spectra with the Elliott formula (Table 1). Also known as 'photo-action spectra'. Red squares and green circles represent MAPbl 3 and MAPbBr $3 \mathrm{NCs} \mathrm{PLQY}$ values, respectively. Lines are guides for the eye. Error bars represent the standard deviation, estimated through repeated measurements. Excitation energy ranges for potential applications of $\mathrm{MAPb}_{3} \mathrm{NCs}$ are included below Figure a. c Absorption (solid line) and photoluminescence (dashed line) of $\mathrm{MAPbBr}_{3}$ and $\mathrm{MAPbl}_{3} \mathrm{NCs}$ in anhydrous toluene solutions. $\mathbf{d}$ Fitting of the $\mathrm{MAPbBr}_{3} \mathrm{NCs}$ absorption spectrum with the Elliott formula, deconvolving the contributions due to free carrier absorption continuum (beige region), Rayleigh scattering (green dashes), and excitonic absorption (bright green region).

Table 1 Parameters for Elliott Model fitting of $\mathrm{MAPbBr}_{3}$ and $\mathrm{MAPbl}_{3} \mathrm{NCs}_{\text {absorption spectra. }}$

\begin{tabular}{llllccc} 
Sample & $\begin{array}{l}\text { Proportionality } \\
\text { constant (a.u.) }\end{array}$ & $\begin{array}{l}\text { Energy } \\
\text { bandgap (eV) }\end{array}$ & $\begin{array}{l}\text { Exciton binding } \\
\text { energy, } \mathbf{E}_{\mathbf{b}}(\mathbf{m e V})\end{array}$ & Linewidth (meV) & $\begin{array}{l}\text { Rayleigh } \\
\text { scattering (a.u.) }\end{array}$ & $\begin{array}{l}\text { Non-parabolicity } \\
(\mathbf{e V}-\mathbf{1})\end{array}$ \\
\hline $\mathrm{MAPb}_{3}$ & $0.37 \pm 0.01$ & $1.73 \pm 0.01$ & $20 \pm 2$ & $59 \pm 3$ & $13 \pm 3$ & $2.1 \pm 0.1$ \\
$\mathrm{MAPbBr}_{3}$ & $0.46 \pm 0.02$ & $2.41 \pm 0.01$ & $36 \pm 1$ & $41 \pm 1$ & $6.8 \pm 0.8$ & $0.10 \pm 0.01$ \\
\hline
\end{tabular}

energy (Table 1), higher than the thermal energy at room temperature $\left(k_{\mathrm{B}} T\right.$ of $26 \mathrm{meV}$ at $\left.T=300 \mathrm{~K}\right)$. Hence, we can infer the presence of stable excitons in $\mathrm{MAPbBr}_{3} \mathrm{NCs}$.

On the other hand, the excitonic feature is much less pronounced in $\mathrm{MAPbI}_{3}$ NCs (Fig. 1c, Supplementary Fig. 5). Weak excitonic contributions are present in the $\mathrm{MAPbI}_{3}$ NCs linear absorption spectrum, likely to reflect strong electron-hole interactions rather than the presence of stable excitons. This is supported by the low-exciton binding energies, equal to or lower than the room temperature thermal energy $\left(k_{\mathrm{B}} T\right.$ of $26 \mathrm{meV}$ ), obtained from the Elliott fitting (Table 1) and consistent with the literature ${ }^{35}$. Despite the differences in the cold carrier dynamics, the dynamics of high-energy excitations are likely to be similar ${ }^{36}$. These excitations produce hot carriers rather than hot excitons due to momentum conservation constraints (i.e., low probability of indirect exciton absorption). Therefore, the early time dynamics is expected to be similar, and differences between $\mathrm{MAPbI}_{3}$ and $\mathrm{MAPbBr}_{3}$ NCs systems are likely to arise after the cooling and exciton formation ${ }^{37,38}$.
The role of hot carrier-induced trapping in perovskites. To delve deeper into the origins of this loss of carriers, we investigated the fate of the hot carriers using femtosecond transient absorption (fs-TA), namely PP, and PPP spectroscopy. Building on previous works that studied the hot carrier dynamics ${ }^{39}$ and transfer ${ }^{40}$, we use the archetypal $\mathrm{MAPbI}_{3} \mathrm{NCs}$ as a model system for ultrafast spectroscopic measurements.

As shown in Fig. 2a, the fs-TA measurement of the $\mathrm{MAPbI}_{3}$ NCs hot carrier cooling dynamics shows the characteristic broadening of the main photobleaching peak ( $\mathrm{PB}$, i.e., positive $\Delta T / T)$, located at $1.70 \mathrm{eV}^{24,37,41,42}$. Following past reports on hybrid and inorganic PNCs, we extracted the hot carrier temperatures and their cooling rate (Fig. $2 \mathrm{a}$ inset $)^{42}$. In agreement with literature ${ }^{42}$, two main cooling regimes are observed: in the low-fluence regime, the cooling is driven by carrier-phonon scattering, with a characteristic lifetime of $\tau_{\text {cooling }}=500 \pm 100 \mathrm{fs}$ in the high-fluence regime, an additional slower cooling mechanism arises, and it is related to the Auger re-heating processes taking place on the picosecond timescale. Hence, to 

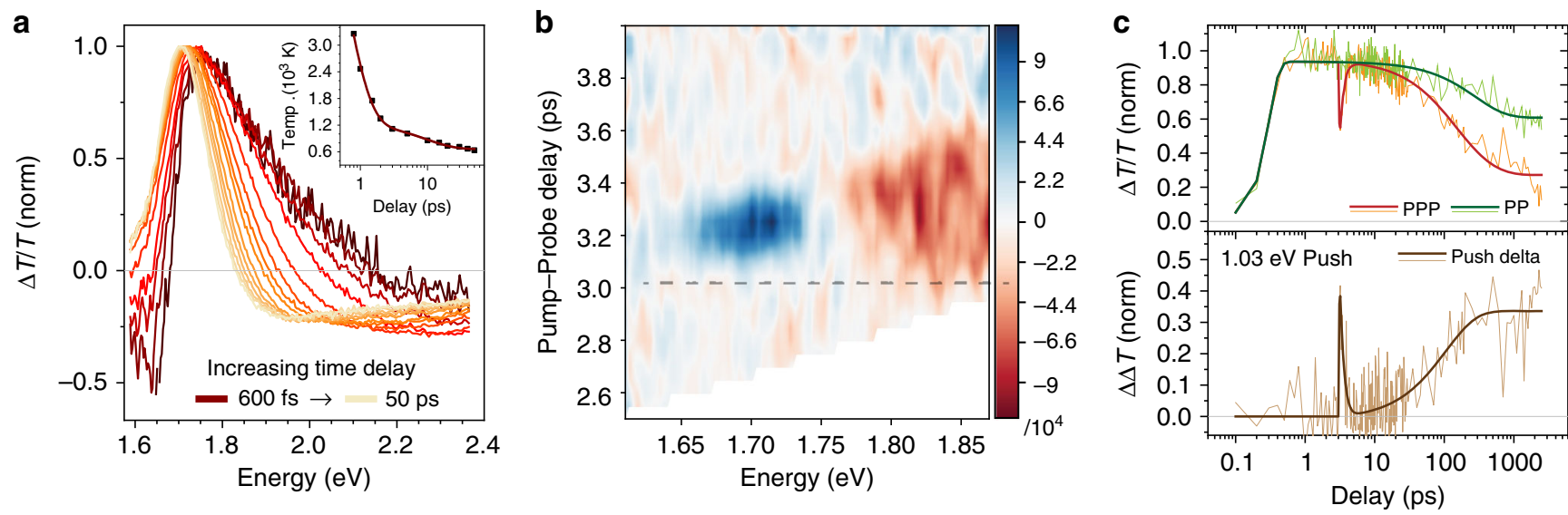

Fig. 2 Time-Resolved Signatures of Hot Carrier-induced Trapping. a Transient Absorption spectrum of MAPbl ${ }_{3} \mathrm{NCs}_{\text {in }}$ anhydrous toluene solutions under intense $(<N>=3.6 \mathrm{e}-\mathrm{h}$ ) high-energy excitation of $3.10 \mathrm{eV}$ (around $1.4 \mathrm{eV}$ in excess of the bandgap). Under this intense excitation, biexponential cooling dynamics is observed and reveals the coexistence of Auger and phonon-mediated relaxation pathways ${ }^{24}$. Inset: Corresponding hot carrier temperatures (black squares), estimated using the Boltzmann model. Multi-exponential fit is represented by the brown line. b Pump-push-probe differential spectrum of $\mathrm{MAPb}_{3} \mathrm{NCs}$ in anhydrous toluene solutions, pumped at $2.07 \mathrm{eV}(10 \mu \mathrm{J} \mathrm{cm}-2,\langle N\rangle=0.88)$, probed over the $1.60-1.90 \mathrm{eV}$ interval, and pushed at $1.03 \mathrm{eV}\left(1 \mathrm{~mJ} \mathrm{~cm}^{-2}\right)$. The black dashed line represents the push time-zero, the positive $\Delta \Delta \mathrm{T}$ signal (blue) implies a decrease in the PB signal, and a negative $\Delta \Delta \mathrm{T}$ signal (red) an increase in the PB signal. c Pump-probe (PP) and pump-push-probe (PPP) kinetics pumped at $1.91 \mathrm{eV}\left(10 \mu \mathrm{Jm}{ }^{-2},<N>=\right.$ 0.8 ) and probed at $1.70 \mathrm{eV}$, with push energy of $1.03 \mathrm{eV}(1 \mathrm{~mJ} \mathrm{~cm}-2)$. Thin lines represent the experimental data, and thick lines are exponential model fits to the data.

avoid complications that could obfuscate analysis and interpretation, we conducted our PPP experiments with low-pump fluence. Raw normalized PPP data are reported in Supplementary Figs. 19-23.

The chirp-corrected differential PPP spectrum for $\mathrm{MAPbI}_{3}$ $\mathrm{NCs}$ with $1.03 \mathrm{eV}$ push energy is shown in Fig. $2 \mathrm{~b}$, as the difference between PP and PPP spectra, i.e., where positive $\Delta \Delta \mathrm{T}$ signal implies decreased $\mathrm{PB}$, and vice versa. The reduced bandedge $\mathrm{PB}$ signal suggests that a fraction of the carriers at the bandedge absorbs the ultrafast push radiation (i.e., free-carrier absorption), before thermalizing and thereby re-heating the carrier distribution. Indeed, the presence of a negative signal at $\sim 1.8 \mathrm{eV}$, blue-shifted with respect to the band-edge, is a signature of the heating of the carrier distribution analogous to the broadening of Fig. 2a. Thus, from PPP measurements, we can directly observe what happens to the hot carriers, and therefore we have the unique opportunity to investigate the effect of excess energy on the carrier dynamics with the presence of a push.

Figure $2 \mathrm{c}$ shows the comparison between the PP and the PPP kinetics of $\mathrm{MAPbI}_{3} \mathrm{NCs}$ with a push providing excess energy, $\delta_{\mathrm{E}}=1.03 \mathrm{eV}$. After the initial pump $(2.07 \mathrm{eV})$, carriers relax to the band-edge and generate an intense $\mathrm{PB}$ signal at the band-edge position (probe $1.70 \mathrm{eV}$ ) 43 . A single-exponential behavior describes the kinetics of the band edge bleaching with lifetime $\tau_{1}=4 \pm 1 \mathrm{~ns}$. Using a push energy of $1.03 \mathrm{eV}\left(1 \mathrm{~mJ} \mathrm{~cm} \mathrm{~cm}^{-2}\right)$ at $3 \mathrm{ps}$ $\mathrm{PP}$ delay time, the push pulse depletes a fraction of the band-edge population $^{39}$. Subsequently, there is a complete recovery (or thermalization) of the excited carriers with a lifetime, $\tau_{\text {thermal }}=$ $450 \pm 100 \mathrm{fs}$, in agreement with the hot carrier relaxation times observed from fs-TA spectroscopy. Although these pushgenerated hot carriers cool down completely (restoring the full PB signal), the subsequent dynamics experience a faster carrier recombination, compared to the case when the push is absent. This is also indicated by the growth of the PPP $\Delta \Delta \mathrm{T}$ kinetic at longer time delays, with an additional lifetime component $(\tau=$ $110 \pm 10$ ps). As shown in Supplementary Figs. 6-8, this effect persists for different pump-push delay times and push fluences.

The hot carrier behavior for a high-energy push $(2.07 \mathrm{eV}$, $60 \mu \mathrm{J} \mathrm{cm}^{-2}$ ) is entirely different and indicates a sudden loss of carriers when the push is absorbed (Supplementary Fig. 9). This sudden disappearance of the carriers could indicate that at higher excess energies other carrier loss mechanisms are present (i.e., higher-lying traps), analogous to previously observed photoionization mechanisms ${ }^{44}$. Moreover, faster recombination dynamics after the push are also observed for the visible-push.

In principle, enhanced Auger recombinations from pushground state absorption processes could cause analogous faster recombination dynamics after the push. For instance, an IR-push $(1.03 \mathrm{eV})$ could generate an additional population from the push ground-state absorption (via two-photon absorption processes), which would be invisible to our detection scheme. In this case, the additional population could enhance the bimolecular and trimolecular (Auger) recombination processes, thereby resulting in faster decay in our PPP kinetics. However, we quantified that this Auger contribution is minute for the case of our IR-push (Supplementary Note 1 and Supplementary Figs. 24-27). On the contrary, we demonstrate that the observed additional lifetime component arises from enhanced monomolecular recombinations (i.e., trapping).

On the other hand, the presence of push ground-state absorption is more significant when employing higher energy pushes (i.e., our visible $2.07 \mathrm{eV}$ push), due to direct competition between the ground-state and the excited-state absorption processes. In this case, our analysis in Supplementary Note 1 shows that enhanced Auger dominates the recombination mechanism after the push. Hence, although the observed positive $\Delta \Delta \mathrm{T}$ upon visible push absorption is a strong indication of possible trapping at high-lying trap sites, the presence of overlapping spectroscopic responses makes their complete disentanglement challenging. Therefore, we limit our further investigation to lower energy excesses.

The unusual behavior observed with increasing excess energy is also reflected in the excitation energy-resolved PP spectroscopy, as shown in Supplementary Fig. 10. When increasing $\delta_{\mathrm{E}}=0.4$ to $0.8 \mathrm{eV}$ by changing the pump energy, the bleaching lifetime reduces from $3.2 \pm 0.6 \mathrm{~ns}$ to $1.9 \pm 0.4 \mathrm{~ns}$, respectively. Notably, when $\delta_{\mathrm{E}}$ is further increased (i.e., at $\delta_{\mathrm{E}}=1.4 \mathrm{eV}$ ), the lifetime does not reduce further, indicating that a certain saturation exists. These results corroborate the PLQY photo-action spectra discussed earlier: when the carriers are residing in higher energy 
states, relaxation and high-energy trapping are observed and provide a possible explanation for the lower PLQY at high $\delta_{\mathrm{E}}$. Meanwhile, PPP spectra for $\mathrm{MAPbBr}_{3}$ NCs show a fundamental difference from that obtained for $\mathrm{MAPbI}_{3} \mathrm{NCs}$, i.e., no additional fast recombination components are detected-see Supplementary Fig. 11. We attribute this difference to arise from the nature of photoexcited species in these two systems. In the less excitonic $\mathrm{MAPbI}_{3} \mathrm{NCs}$ (dominated free carriers), the IR push can readily excite the free carriers, thus affecting the whole photobleaching band (Supplementary Figs. 12 and 14). This is in contrast with the more excitonic $\mathrm{MAPbBr}_{3} \mathrm{NCs}$, where the differential PPP has a derivative-like shape, suggesting that inherent limitations of the PPP technique-possibly conceal the spectroscopic signatures of hot carrier-induced trapping (Supplementary Figs. 13, 14). Nonetheless, the hot carrier-induced trapping is still present in $\mathrm{MAPbBr}_{3} \mathrm{NCs}$-evident from the PLQY photo-action spectra.

\section{Discussion}

Herein, we propose a possible explanation for the observed dynamics in the PPP spectrum (IR push) that involves the interaction of hot carriers with shallow traps. Thus, while cold carriers mainly interact with deep traps as observed by nonunity PLQY values at very low-excess energies, we propose that the push-induced heating of the carriers opens additional trapping channels. Our hypothesis is based on the two main spectroscopic features arising from the PPP: (1) the ultrafast relaxation leading to a full recovery of the $\mathrm{PB}$ signal after the push pulse; and (2) the enhanced recombination induced by the push (Fig. 2c). These observations suggest that the hot carriers could promote the formation of shallow trapped carrier states, where one carrier (either the electron or the hole) gets trapped at a shallow trap site but still contributes to the signal in terms of defect stimulated emission. This would explain the observed faster recombination after the push as increased non-radiative recombination caused by shallow trap states ${ }^{45,46}$. Our results suggest that this trapping takes place on the same timescale as the cooling. Analogous considerations on HC trapping were reported in past studies on $\mathrm{CdSe}^{47}$ and recent studies on $\mathrm{MAPbI}_{3}$ thin films ${ }^{19}$. However, although the presence of shallow traps in PNCs is already well-known and provides a good explanation for their PL dynamics ${ }^{48-51}$, the interactions of hot carriers with these traps have yet to be understood.

While PNCs surface chemistry shows some differences compared with that of conventional II-VI quantum dots (QDs) (e.g., $\mathrm{CdSe}$ ), they both share similarities such as the presence of undercoordinated surface metal atoms, which act as carrier $\operatorname{traps}^{29,52}$. Hence, we use the well-established understanding of surface traps in II-VI QDs as our starting point. The pivotal role played by shallow traps in CdSe QDs was reported by Scholes and Kambhampati in their seminal papers, where the charge-trapping process to shallow defect sites is described using the Marcus (or Marcus-Jortner) charge transfer model ${ }^{45,46}$. The corollary of these works is that the trapping/detrapping equilibrium governs the population of charged dots (i.e., the number of trapped carriers in the ensemble), and this limits the PLQY due to faster nonradiative recombination for trapped carriers $17,32,49$. Notably, the same trapping dynamics has been reported for cold carriers in $\mathrm{MAPbBr}_{3}$ and $\mathrm{MAPbI}_{3} \mathrm{NCs}$, thus confirming the similar role played by surface traps ${ }^{51,53-55}$. Such an equilibrium-usually dictated by many factors, e.g., the temperature, the energetic levels of the traps-can be perturbed by the increased trapping rates for the hotter carriers.

Based on our qualitative understanding so far, we developed a model to describe the hot carriers and how they interact with these trap states in $\mathrm{MAPbI}_{3} \mathrm{NCs}$ (Fig. 3a). Briefly, for $\mathrm{MAPbI}_{3}$
NCs the population dynamics of both the free carrier $(N)$ and shallow-trapped carriers $\left(N_{\mathrm{T}}\right)$ states can be described by the following expressions:

$$
\frac{\mathrm{d} N(t)}{\mathrm{d} t}=-k_{2} N^{2}(t)-\int_{0}^{\infty} k_{\mathrm{T}}(\epsilon) n(\epsilon, t) \mathrm{d} \epsilon+e^{\Delta G_{0} / k_{\mathrm{B}} T} \int_{0}^{\infty} k_{\mathrm{T}}(\epsilon) n_{\mathrm{T}}(\epsilon, t) \mathrm{d} \epsilon,
$$

$$
\frac{\mathrm{d} N_{\mathrm{T}}(t)}{\mathrm{d} t}=-k_{\mathrm{tr}} N_{\mathrm{T}}(t)+\int_{0}^{\infty} k_{\mathrm{T}}(\epsilon) n(\epsilon, t) \mathrm{d} \epsilon-e^{\Delta G_{0} / k_{\mathrm{B}} T} \int_{0}^{\infty} k_{\mathrm{T}}(\epsilon) n_{\mathrm{T}}(\epsilon, t) \mathrm{d} \epsilon .
$$

Here, $\epsilon$ is the carrier energy axis; $k_{2}$ is the bimolecular recombination rate; and $k_{\mathrm{tr}}$ is the non-radiative recombination rate from the trap potential; $n(\epsilon, t)$ is the thermally equilibrated carrier distribution with temperature $T(\mathrm{t})$ in a parabolic band system; $k_{\mathrm{T}}(\epsilon)$ is the population exchange rate between the two potential energy surfaces as a function of carrier energy; and $\Delta G_{0}$ is the Gibbs free energy (i.e., the difference in energy between reactant and product energy surfaces at the equilibrium). The second and third terms in Eqs. (1) and (2) describe the population exchanges between the parabolas. We assume the phenomenological carrier temperature to be $T(t)=300 \mathrm{~K}+T_{0} \exp \left(-t / \tau_{\mathrm{c}}\right)$, where $T_{0}=\delta_{\mathrm{E}} / k_{\mathrm{B}}$ is the carrier temperature post-excitation (assuming the almost instantaneous formation of Boltzmann distribution), that cools down with cooling time constant $\tau_{\mathrm{c}}{ }^{24}$. In agreement with Elliott fitting results, we accounted for the more excitonic nature of the recombination in $\mathrm{MAPbBr}_{3} \mathrm{NCs}$ by replacing the carrier population term presented in Eqs. (1) and (3). Specifically, the radiative bimolecular recombination term is replaced by a monomolecular radiative recombination term with rate $k_{\mathrm{r}}$, [i.e., $-k_{2} N^{2}(t) \rightarrow-k_{\mathrm{r}} N(t)$ ]. The full description of the model is given in Supplementary Note 2. The PLQY of the system with an initial photoexcited carrier population $N_{0}$, can be calculated as:

$$
\operatorname{PLQY}\left(\delta_{\mathrm{E}}\right)=\frac{k_{2} \int_{0}^{\infty} N^{2}(t) \mathrm{d} t}{N_{0}} .
$$

It is worth noting that in this model the hot carriers have two competing pathways (Fig. $3 \mathrm{~b}, \mathrm{c}$ ): (1) relaxation to the band-edge, in accordance with the literature on $\mathrm{MAPbI}_{3}{ }^{56}$; and (2) being trapped at the traps' potentials. The model also describes how the trapping of hotter carriers occurs with a higher driving force, $-\left(\Delta G_{0}+\delta_{\mathrm{E}}\right)$, compared with the cold carriers. Therefore, trapping rates increase and become more dominant as carriers get hotter (due to the $\delta_{\mathrm{E}}$ term), thereby leading to a higher number of trapped carriers within the PNCs.

This can explain the downward PLQY dependence with increasing excess energy, and the effects are clearly illustrated in Fig. $3 \mathrm{~d}$, e. These findings have very strong implications on the impact of the traps on hot carriers: the defect tolerance ${ }^{29}$ declines when carriers are more energetic. In other words, these traps become activated under high-energy excitation conditions, a hallmark of applications such as light-emitting devices and photovoltaics.

To gain a deeper insight into the surface chemistry of the defects in PNCs, we exchanged the native alkylamine/oleic acid ligand couple with trioctylphosphine oxide (TOPO). TOPO has previously been shown to passivate and improve the emissive properties of bulk $\mathrm{MAPbI}_{3}$ thin films ${ }^{57,58}$. $\mathrm{MAPbI}_{3}$ and $\mathrm{MAPbBr}_{3}$ share common defect chemistry, and a remarkably defect tolerant electronic structure (i.e., the ability to retain the electronic structure even with large defect concentrations) $7,27,29,59$. For instance in $\mathrm{MAPbI}_{3}$, many first-principle calculations, and experiments (PL, XPS) show that most of the stable defects (i.e., vacancies $\mathrm{V}_{\mathrm{MA}}, \mathrm{V}_{\mathrm{I}}, \mathrm{V}_{\mathrm{Br}}$, and interstitials $\mathrm{MA}_{\mathrm{i}}, \mathrm{Pb}_{\mathrm{i}}$ ) introduce shallow traps, while deep electron/hole traps are associated with 
a

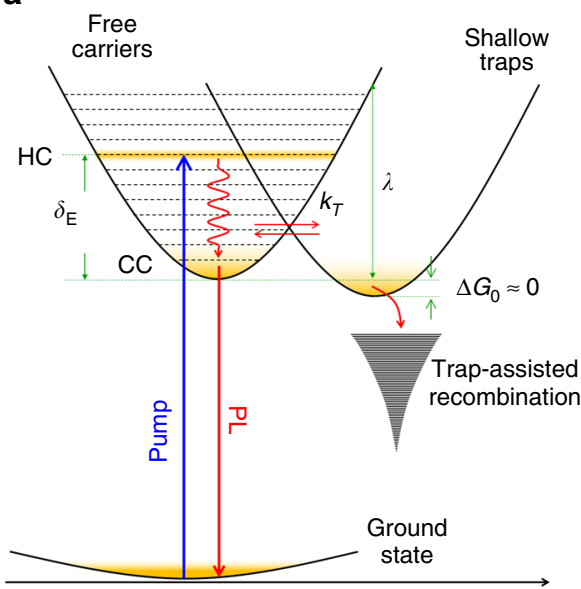

Nuclear coordinate

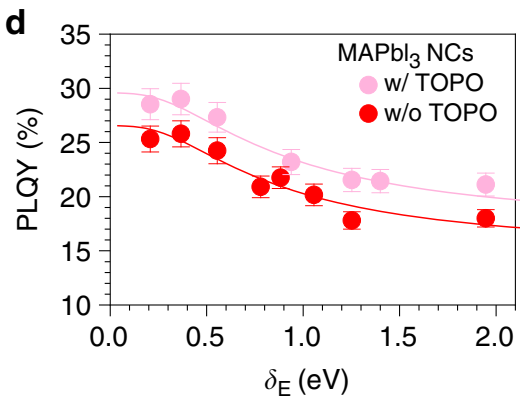

b

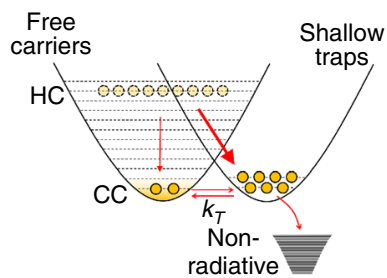

High excess excitation energy

C

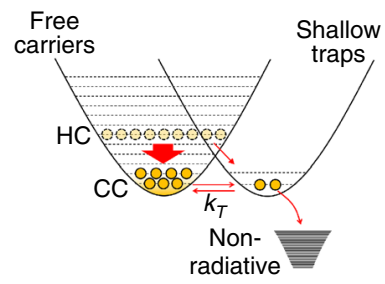

Low excess excitation energy

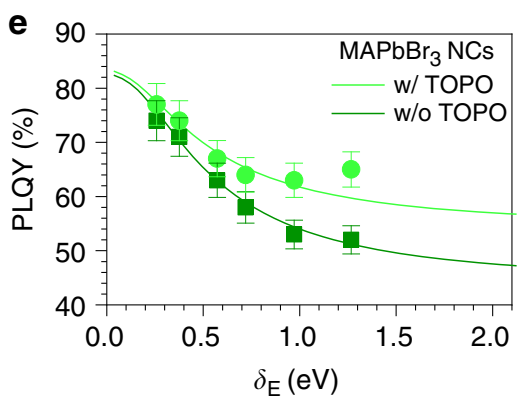

Fig. 3 Phenomenological model of hot carrier-induced trapping. a Model schematic describing the interaction between carriers and shallow traps in PNCs. Free and trapped carriers are involved in a thermal equilibrium, whose barrier is dictated by the combination of reorganization energy, $\lambda$, and trapping free energy $-\Delta G_{0}$. $\mathbf{b}$ The high-excess energy $\left(\delta_{\mathrm{E}}\right)$ for the carriers increases the trapping rate and hence results in a higher number of trapped carriers, i.e., lower PLQY. c Vice versa, lower $\delta_{\mathrm{E}}$ results in lower trapping rate and thus results in a lower number of trapped carriers, i.e., higher PLQY. HC and $\mathrm{CC}$ indicate the hot carriers and cold carriers, respectively. Model fitting of the photo-action spectra, for (d) $\mathrm{MAPbl}_{3} \mathrm{NCs}(\mathbf{e})$ and $\mathrm{MAPbBr}_{3} \mathrm{NCs}$. The light-colored dots represent TOPO ligand-exchanged PNCs, while dark dots represent pristine PNCs. Error bars represent the standard deviation, estimated through repeated measurements. The corresponding colored lines represent the model fitting.

the less abundant interstitial iodine $\left(\mathrm{I}_{\mathrm{i}}\right)$ defects and lead vacancies $\left(\mathrm{V}_{\mathrm{Pb}}\right)^{6,7,59}$. Furthermore, the electronic structure was found to be particularly stable with respect to the formation of charge compensating defect pairs (e.g., $\mathrm{V}_{\mathrm{MA}}$ and $\left.\mathrm{V}_{\mathrm{I}}\right)^{29}$. According to the Green taxonomy, the $\mathrm{P}=\mathrm{O}$ moiety makes TOPO a neutral donor (L-type ligand) that can efficiently passivate under-coordinate metal $(\mathrm{Pb})$ atoms from these vacancies through Lewis adduct formation $52,60,61$.

Our model quantifies the hot carrier-trap interaction in terms of the two most important parameters: $H_{\mathrm{s}}$, the electronic coupling of the carriers to the defects; and $\lambda$, the reorganization energy associated with the trap-induced structural distortions ${ }^{46}$. The values of $H_{s}$ and $\lambda$ (Supplementary Table 1) are consistent with previous reports of CdSe $\mathrm{QDs}^{46}$, and they are reduced when TOPO-passivation is applied. As shown in Fig. 3c, TOPOpassivation is beneficial for PNCs, providing overarching improvements in the PLQY of $\mathrm{MAPbI}_{3} \mathrm{NCs}$, and mitigating the loss in PLQY at high-excess energies for $\mathrm{MAPbBr}_{3} \mathrm{NCs}\left(\delta_{\mathrm{E}}>\right.$ $0.2 \mathrm{eV}$ ). As the excitation energy is closer to the band-edge, shallow traps are essentially optically-inactive, and the observed improvement is smaller. With increasing excess energy, improper passivation of the shallow traps, or the lack thereof, consistently leads to a loss of carriers relaxing from high-energy states. Our PPP results corroborate these findings and are reported in Supplementary Figs. 16, 17. Here, the comparison between differential PPP kinetics for pristine and ligand-exchanged $\mathrm{MAPbI}_{3}$ evidences how the additional TOPO passivation results in lower carrier losses to both ligands and shallow traps sites. Therefore, these experiments confirm that the observed reduction of the PLQY is related to the surface passivation of the perovskite NCs. Specifically, traps that are supposedly benign or 'inactive' for the cold carriers, and whose passivation is generally not considered as necessary ${ }^{7}$, become active under the energy (or the 'heat') of the hot carriers.

Hot carrier-induced trapping processes provide an additional and alternative cooling pathway that intuitively should speed up overall hot carrier cooling times. However, a direct comparison between PPP data for pristine and ligand-exchange $\mathrm{MAPbI}_{3} \mathrm{NCs}$ (Supplementary Fig. 16b) indicates that while the long-time recombination dynamics are affected by the passivation, the cooling dynamics are unchanged within our time resolution. Similar results for the cooling were recently reported by Harel et al. for bulk MAPI and Bakulin et al. for other PNCs ${ }^{19,62}$. Hence, we speculate that defects passivated by TOPO do not significantly affect the electron-phonon coupling, and therefore do not significantly speed up the cooling dynamics.

Nevertheless, a proper passivation is crucial for HC solar cells, ASE and lasing applications. Indeed, as suggested by the passivation effect of HC temperatures (Supplementary Fig. 16e), HC carrier losses could still affect these applications in terms of their efficiencies (i.e., reducing the number of carriers available for extraction or photon conversion, respectively) ${ }^{4}$. Future efforts in 
studying the passivation effects on these applications are needed. Moreover, the increased knowledge of HC-induced trapping processes provides a different perspective on the many unanswered questions in the perovskite field, e.g., polaron dynamics, hot carrier cooling and extraction, and multiple exciton generation (MEG). Zhu and co-workers have provided an excellent picture of the hot polaron formation dynamics, as an alternative relaxation pathway to carrier-LO phonon scattering ${ }^{62}$. Notably, the study of hot polarons is usually conducted at low fluences below the Mott Density, which is also the regime of interest in this study ${ }^{63}$. It is therefore interesting to note that these hot carrier-induced trapping processes might complete the picture of large polarons $s^{64}$ in PNCs, and could help rationalize the absence of hot PL in $\mathrm{CsPbBr}_{3}{ }^{65,66}$.

In conclusion, we uncover the detrimental effects of hot carrier traps on the PLQY of $\mathrm{MAPbBr}_{3}$ and $\mathrm{MAPbI}_{3}$ PNCs. Our excitation energy-dependent PLQY and PPP measurements demonstrate that when hot carriers with moderate energies in excess of the band-gap are present, shallow trap sites are activated. This startling activity of shallow defects, previously deemed as innocuous, suggests a reconsideration of the defect-tolerance in these systems. We use a phenomenological model to explain why hotter carriers experience increased trapping rates to low-energy shallow traps, thereby transforming these benign defects into malignant ones. Our model also reveals how the electronic coupling of the carriers to the traps and its associated reorganization energy can be changed by passivating the PNCs' surface. We experimentally support this by performing a ligand exchange (with TOPO as the ligand) to passivate these traps, leading to tangible improvements in the PLQY excitation profile. However, the adopted procedure is but one of many possible strategies that can be employed, and we are confident that these insights will help pave the way towards highly efficient PNC devices. Our findings provide a more holistic understanding of 'defect-tolerant' PNCs and have potential implications on how the future photophysical studies of high-energy carriers can be interpreted.

\section{Methods}

Materials. Lead Bromide, $\mathrm{PbBr}_{2}$ and Lead Iodide (99.999\%, trace metals basis), oleylamine (>98\%; OlAm), n-octylamine (99\%; OctAm), oleic acid ( $90 \%$ : OlAc), N,N-dimethylformamide, (99.8\% anhydrous; DMF), acetonitrile ( $99.8 \%$ anhydrous; ACN), toluene (anhydrous, 99.7\% GC), trioctylphenylphosphine (99\% Reagent Plus; TOPO $\left.^{\circledR}\right)$ and benzyl alcohol $(99.5 \%, \mathrm{BnOH})$ were purchased from SigmaAldrich. Methylammonium bromide (MABr) and methylammonium iodide (MAI) were purchased from Greatcell Solar Material. The chemicals were used without further purification.

Synthesis of $\mathbf{M A P b B r}_{\mathbf{3}} \mathbf{N C s}$. The precursor solution was prepared in $2 \mathrm{~mL}$ DMF, dissolving completely $0.04 \mathrm{mmol}$ of $\mathrm{PbBr}_{2}(146.8 \mathrm{mg})$ and $0.04 \mathrm{mmol}$ of $\mathrm{MABr}$ $(44.8 \mathrm{mg})$. After the complete dissolution of the salts by bath sonication, the PNCs were synthesized by swift injection of $200 \mu \mathrm{L}$ of precursor solution into an antisolvent solution under vigorous stirring. The antisolvent solution was prepared adding $20 \mu \mathrm{L} \mathrm{OctAm}, 1000 \mu \mathrm{L} \mathrm{OlAc}$, and $700 \mu \mathrm{L}$ to $5 \mathrm{~mL}$ of toluene. After the swift injection, a yellow/green suspension was obtained. The purification of the crude followed previously a previously published synthesis (ref. ${ }^{18}$, main text) and comprised two centrifugation-redispersion steps at $12,000 \mathrm{rpm}$ and $4000 \mathrm{rpm}$, respectively. Subsequently, the precipitated nanocrystals were dispersed in $1 \mathrm{~mL}$ of anhydrous toluene and stored in at $4^{\circ} \mathrm{C}$.

Synthesis of $\mathbf{M A P b}_{\mathbf{3}} \mathbf{N C s}$. The precursor solution was prepared by dissolving completely $0.1 \mathrm{mmol}$ of $\mathrm{PbI}_{2}(45.9 \mathrm{mg})$ and $0.1 \mathrm{mmol}$ of MAI $(15.9 \mathrm{mg})$ into a solution of $2 \mathrm{~mL}$ ACN, $20 \mu \mathrm{L}$ OlAm, $0.20 \mathrm{~mL}$ OlAc. The full precursor solution was slowly injected (dropwise addition) to $10 \mathrm{~mL}$ toluene vigorously stirred under an ambient atmosphere. The purification of the crude comprised one centrifugation/ redispersion step at $8000 \mathrm{rpm}$ and a second centrifuge step at $4000 \mathrm{rpm}$ to remove the bulkier nanoparticles. The supernatant was then stored in a nitrogen glovebox for further usage.

X-ray diffraction (XRD). XRD measurements on drop cast thin film samples were performed using Rigaku SmartLab High-Resolution X-ray diffractometer with $\mathrm{Cu}$ $\mathrm{Ka}$ (wavelength of $1.5418 \AA$ ) X-ray source. The measurement was done in the general medium resolution PB/PSA (theta-2 theta) mode scanning from 10 to 45 degrees with steps of 0.02 degrees.

Linear optical spectroscopy. UV-Vis spectra were recorded by using a Shimadzu UV-3600 Plus spectrometer, in the range $200-900 \mathrm{~nm}$. The PL spectra were measured with a FluoroLog (Jobin-Yvon) fluorimeter. Diluted sample solutions in anhydrous toluene were contained in quartz cuvettes.

Photoluminescence quantum yield (PLQY). PLQY measurements were performed using a Horiba Jobin-Yvon Fluorolog system equipped with iHR320 monochromator, coupled with a photomultiplier tube and a spectrally calibrated Spectralon-coated integrating sphere (Quanta-Phi). Excitation energy was varied by selecting different components of a Xe lamp emission with a monochromator. Diluted solutions of the samples (OD $<0.1)$ were contained in a quartz $1 \mathrm{~cm} \times 1 \mathrm{~cm}$ cuvette. A relative error of about $5 \%$ on measured PLQY values was estimated by repeated measurements.

Femtosecond transient absorption (fs-TA) spectroscopy. fs-TA measurements were performed using a Helios spectrometer (Ultrafast Systems, LLC). The pump pulse was a frequency-doubled fundamental emission $(400 \mathrm{~nm}, 3.1 \mathrm{eV}$ pulse) generated from a Coherent Legend $(150 \mathrm{fs}, 1 \mathrm{kHz}, 800 \mathrm{~nm})$ regenerative amplifier. Excitation energy-resolved pump pulses were generated from an optical parametric amplifier (Light Conversion TOPAS-C). The white light continuum probe pulse (in the range from 420 to $780 \mathrm{~nm}$ ) was generated by focusing the residual of the regenerative amplifier's fundamental $800 \mathrm{~nm}$ laser pulses into either a $2 \mathrm{~mm}$ sapphire crystal (for visible range). Multichannel acquisition of the entire spectrum was achieved using a cMOS detector. Samples were contained into $2 \mathrm{~mm}$ cuvette and vigorously stirred using a Magnetic Stirrer (Ultrafast Systems, LLC) to avoid photocharging effects. The calculation of the absorption cross section for $\mathrm{MAPbI}_{3}$ NCs is reported in Supplementary Fig. 15.

Pump-Push-Probe (PPP) spectroscopy. A home-built setup in transmission geometry was used to perform PPP spectroscopy measurements. The output from a Coherent Libra regenerative amplifier $(1 \mathrm{kHz}, 800 \mathrm{~nm}, 50 \mathrm{fs})$ was split to three beams, two to pump two Coherent OPerA-Solo optical parametric amplifiers. The remaining $800 \mathrm{~nm}$ fundamental beam used for WLC generation $(450$ to $780 \mathrm{~nm})$ was attenuated with a $750 \mathrm{~nm}$ short-pass filter (estimated probe fluence of $\sim 0.14 \mu \mathrm{J} \mathrm{cm}^{-2}$ at $\sim 1.63 \mathrm{eV}$ ). The first OPA was used to generate the pump pulse train $(1.91 \mathrm{eV}, 2.07 \mathrm{eV})$, while the second OPA was used to generate the push pulse train $(1.03 \mathrm{eV}, 2.07 \mathrm{eV})$. In the experiments on $\mathrm{MAPbBr}_{3} \mathrm{NCs}$, the $3.1 \mathrm{eV}$ pump was generated by the frequencydoubling of the residual fundamental output using a BBO crystal. The pump was chopped at $83 \mathrm{~Hz}$, in combination with a modulated push, the PP, and PPP signals were obtained separately and averaged across at least 3 scans. Taking the difference yields the push- induced signal (described in the main text). Both push and probe pulse trains were mechanically delayed by precision delay stages and the probe was collected by a spectrometer (Princeton Instruments Acton SP-2300i) coupled to a PMT point detector and collected by the computer through an SRS 830 lock-in amplifier. Normalized data is presented in the manuscript, while raw data is reported in the SI. The stability of the samples during PPP experiments is given in Supplementary Fig. 18.

\section{Data availability}

The data that support the findings of this study are openly available in DR-NTU (Data) at: https://doi.org/10.21979/N9/EGH6UI. Data is also available from the Corresponding Author upon reasonable request.

Received: 23 October 2019; Accepted: 21 April 2020; Published online: 01 June 2020

\section{References}

1. Stranks, S. D. \& Snaith, H. J. Metal-halide perovskites for photovoltaic and light-emitting devices. Nat. Nanotechnol. 10, 391 (2015).

2. Green, M. A., Ho-Baillie, A. \& Snaith, H. J. The emergence of perovskite solar cells. Nat. Photonics 8, 506 (2014).

3. Tan, Z.-K. et al. Bright light-emitting diodes based on organometal halide perovskite. Nat. Nanotechnol. 9, 687 (2014)

4. Xing, G. et al. Low-temperature solution-processed wavelength-tunable perovskites for lasing. Nat. Mater. 13, 476 (2014).

5. Giovanni, D. et al. Ultrafast long-range spin-funneling in solution-processed Ruddlesden-Popper halide perovskites. Nat. Commun. 10, 3456 (2019).

6. Meggiolaro, D. \& De Angelis, F. First-principles modeling of defects in lead halide perovskites: best practices and open issues. ACS Energy Lett. 3, 2206-2222 (2018) 
7. Meggiolaro, D. et al. Iodine chemistry determines the defect tolerance of leadhalide perovskites. Energy Environ. Sci. 11, 702-713 (2018).

8. Ran, C., Xu, J., Gao, W., Huang, C. \& Dou, S. Defects in metal triiodide perovskite materials towards high-performance solar cells: origin, impact, characterization, and engineering. Chem. Soc. Rev. 47, 4581-4610 (2018).

9. Hutter, E. M., Eperon, G. E., Stranks, S. D. \& Savenije, T. J. Charge carriers in planar and meso-structured organic-inorganic perovskites: mobilities, lifetimes, and concentrations of trap states. J. Phys. Chem. Lett. 6, 3082-3090 (2015).

10. Leijtens, T. et al. Electronic properties of meso-superstructured and planar organometal halide perovskite films: charge trapping, photodoping, and carrier mobility. ACS Nano 8, 7147-7155 (2014).

11. Motti, S. G. et al. Controlling competing photochemical reactions stabilizes perovskite solar cells. Nat. Photonics 13, 532-539 (2019).

12. Motti, S. G. et al. Defect activity in metal-halide perovskites. Adv. Mater. 0, 1901183 (2019).

13. Chen, B., Rudd, P. N., Yang, S., Yuan, Y. \& Huang, J. Imperfections and their passivation in halide perovskite solar cells. Chem. Soc. Rev. 48, 3842-3867 (2019).

14. Brandt, R. E. et al. Searching for "defect-tolerant" photovoltaic materials: combined theoretical and experimental screening. Chem. Mater. 29, 4667-4674 (2017).

15. Kurchin, R. C., Gorai, P., Buonassisi, T. \& Stevanović, V. Structural and chemical features giving rise to defect tolerance of binary semiconductors. Chem. Mater. 30, 5583-5592 (2018).

16. del Valle, J. C. \& Catalán, J. Kasha's rule: a reappraisal. Phys. Chem. Chem. Phys. 21, 10061-10069 (2019).

17. Li, B., Brosseau, P. J., Strandell, D. P., Mack, T. G. \& Kambhampati, P. Photophysical action spectra of emission from semiconductor nanocrystals reveal violations to the vavilov rule behavior from hot carrier effects. J. Phys. Chem. C 123, 5092-5098 (2019).

18. Bretschneider, S. A., Laquai, F. \& Bonn, M. Trap-free hot carrier relaxation in lead-halide perovskite films. J. Phys. Chem. C 121, 11201-11206 (2017).

19. Jiang, X., Hoffman, J., Stoumpos, C. C., Kanatzidis, M. G. \& Harel, E. Transient sub-band-gap states at grain boundaries of $\mathrm{CH} 3 \mathrm{NH} 3 \mathrm{PbI} 3$ perovskite act as fast temperature relaxation centers. ACS Energy Lett. 4, 1741-1747 (2019).

20. Lan, X. et al. Passivation using molecular halides increases quantum dot solar cell performance. Adv. Mater. 28, 299-304 (2016).

21. Kirkwood, N. et al. Finding and fixing traps in II-VI and III-V colloidal quantum dots: the importance of Z-type ligand passivation. J. Am. Chem. Soc. 140, 15712-15723 (2018).

22. Jang, Y. et al. Interface control of electronic and optical properties in IV-VI and II-VI core/shell colloidal quantum dots: a review. Chem. Comm. 53, 1002-1024 (2017).

23. Li, M. et al. Low threshold and efficient multiple exciton generation in halide perovskite nanocrystals. Nat. Commun. 9, 4197 (2018).

24. Li, M., Fu, J., Xu, Q. \& Sum, T. C. Slow hot-carrier cooling in halide perovskites: prospects for hot-carrier solar cells. Adv. Mater. 0, 1802486 (2019).

25. Chen, W. et al. Giant five-photon absorption from multidimensional coreshell halide perovskite colloidal nanocrystals. Nat. Commun. 8, 15198 (2017).

26. Niesner, D. et al. Persistent energetic electrons in methylammonium lead iodide perovskite thin films. J. Am. Chem. Soc. 138, 15717-15726 (2016).

27. Akkerman, Q. A., Rainò, G., Kovalenko, M. V. \& Manna, L. Genesis, challenges and opportunities for colloidal lead halide perovskite nanocrystals. Nat. Mater. 17, 394-405 (2018).

28. Protesescu, L. et al. Nanocrystals of cesium lead halide perovskites (CsPbX3, $\mathrm{X}=\mathrm{Cl}, \mathrm{Br}$, and I): novel optoelectron $-\mathrm{ic}$ materials showing bright emission with wide color gamut. Nano Lett. 15, 3692-3696 (2015).

29. Steirer, K. X. et al. Defect tolerance in methylammonium lead triiodide perovskite. ACS Energy Lett. 1, 360-366 (2016).

30. Veldhuis, S. A. et al. Benzyl alcohol-treated $\mathrm{CH}_{3} \mathrm{NH}_{3} \mathrm{PbBr}_{3}$ nanocrystals exhibiting high luminescence, stability, and ultralow amplified spontaneous emission thresholds. Nano Lett. 17, 7424-7432 (2017).

31. Zhang, F. et al. Colloidal synthesis of air-stable $\mathrm{CH}_{3} \mathrm{NH}_{3} \mathrm{PbI}_{3}$ quantum dots by gaining chemical insight into the solvent effects. Chem. Mater. 29, 3793-3799 (2017).

32. Kambhampati, P. Hot exciton relaxation dynamics in semiconductor quantum dots: radiationless transitions on the nanoscale. J. Phys. Chem. C 115, 22089-22109 (2011)

33. Saba, M. et al. Correlated electron-hole plasma in organometal perovskites. Nat. Commun. 5, 5049 (2014).

34. Elliott, R. J. Intensity of optical absorption by excitons. Phys. Rev. 108, 1384-1389 (1957).

35. Chen, X., Lu, H., Yang, Y. \& Beard, M. C. Excitonic effects in methylammonium lead halide perovskites. J. Phys. Chem. Lett. 9, 2595-2603 (2018).

36. Sarritzu, V. et al. Perovskite excitonics: primary exciton creation and crossover from free carriers to a secondary exciton phase. Adv. Opt. Mater. 6, 1700839 (2018).
37. Yang, Y. et al. Observation of a hot-phonon bottleneck in lead-iodide perovskites. Nat. Photonics 10, 53 (2015).

38. Ghosh, T., Aharon, S., Etgar, L. \& Ruhman, S. Free carrier emergence and onset of electron-phonon coupling in methylammonium lead halide perovskite films. J. Am. Chem. Soc. 139, 18262-18270 (2017).

39. Hopper, T. R. et al. Ultrafast intraband spectroscopy of hot-carrier cooling in lead-halide perovskites. ACS Energy Lett. 3, 2199-2205 (2018).

40. Lim, S. S. et al. Hot carrier extraction in $\mathrm{CH}_{3} \mathrm{NH}_{3} \mathrm{PbI}_{3}$ unveiled by pumppush-probe spectroscopy. Sci. Adv. 5, eaax3620 (2019).

41. Fu, J. et al. Hot carrier cooling mechanisms in halide perovskites. Nat. Commun. 8, 1300 (2017).

42. Li, M. et al. Slow cooling and highly efficient extraction of hot carriers in colloidal perovskite nanocrystals. Nat. Commun. 8, 14350 (2017).

43. Sum, T. C. et al. Spectral features and charge dynamics of lead halide perovskites: origins and interpretations. Acc. Chem. Res. 49, 294-302 (2016).

44. McGuire, J. A. et al. Spectroscopic signatures of photocharging due to hotcarrier transfer in solutions of semiconductor nanocrystals under lowintensity ultraviolet excitation. ACS Nano 4, 6087-6097 (2010).

45. Mooney, J., Krause, M. M., Saari, J. I. \& Kambhampati, P. Challenge to the deep-trap model of the surface in semiconductor nanocrystals. Phys. Rev. B 87, 081201 (2013)

46. Jones, M., Lo, S. S. \& Scholes, G. D. Quantitative modeling of the role of surface traps in CdSe/CdS/ZnS nanocrystal photoluminescence decay dynamics. Proc. Natl Acad. Sci. USA 106, 3011 (2009).

47. Sewall, S. L. et al. State-resolved studies of biexcitons and surface trapping dynamics in semiconductor quantum dots. J. Chem. Phys. 129, 084701 (2008).

48. Minotto, A. et al. Role of core-shell interfaces on exciton recombination in CdSe-CdxZn1-xS quantum dots. J. Phys. Chem. C 118, 24117-24126 (2014).

49. Righetto, M., Minotto, A. \& Bozio, R. Bridging energetics and dynamics of exciton trapping in core-shell quantum dots. J. Phys. Chem. C 121, 896-902 (2017).

50. Rabouw, F. T. et al. Delayed exciton emission and its relation to blinking in CdSe quantum dots. Nano Lett. 15, 7718-7725 (2015).

51. Diroll, B. T., Guo, P. \& Schaller, R. D. Unique optical properties of methylammonium lead iodide nanocrystals below the bulk tetragonalorthorhombic phase transition. Nano Lett. 18, 846-852 (2018).

52. Boles, M. A., Ling, D., Hyeon, T. \& Talapin, D. V. The surface science of nanocrystals. Nat. Mater. 15, 141 (2016).

53. Chirvony, V. S. et al. Delayed luminescence in lead halide perovskite nanocrystals. J. Phys. Chem. C 121, 13381-13390 (2017).

54. Chirvony, V. S. \& Martínez-Pastor, J. P. Trap-limited dynamics of excited carriers and interpretation of the photoluminescence decay kinetics in metal halide perovskites. J. Phys. Chem. Lett. 9, 4955-4962 (2018).

55. Shi, H., Zhang, X., Sun, X., Chen, R. \& Zhang, X. Direct and indirect recombination and thermal kinetics of excitons in colloidal all-inorganic lead halide perovskite nanocrystals. J. Phys. Chem. C 123, 19844-19850 (2019).

56. Filip, M. R., Verdi, C. \& Giustino, F. GW band structures and carrier effective masses of $\mathrm{CH}_{3} \mathrm{NH}_{3} \mathrm{PbI}_{3}$ and hypothetical perovskites of the type $\mathrm{APbI}_{3}: \mathrm{A}=$ $\mathrm{NH}_{4}, \mathrm{PH}_{4}, \mathrm{AsH}_{4}$, and $\mathrm{SbH}_{4}$. J. Phys. Chem. C 119, 25209-25219 (2015).

57. $\mathrm{Xu}, \mathrm{W}$. et al. Rational molecular passivation for high-performance perovskite light-emitting diodes. Nat. Photonics 13, 418-424 (2019).

58. deQuilettes, D. W. et al. Photoluminescence lifetimes exceeding $8 \mu$ s and quantum yields exceeding $30 \%$ in hybrid perovskite thin films by ligand passivation. ACS Energy Lett. 1, 438-444 (2016).

59. Yin, W.-J., Shi, T. \& Yan, Y. Unusual defect physics in $\mathrm{CH} 3 \mathrm{NH} 3 \mathrm{PbI} 3$ perovskite solar cell absorber. Appl. Phys. Lett. 104, 063903 (2014).

60. Green, M. L. H. A new approach to the formal classification of covalent compounds of the elements. J. Organomet. Chem. 500, 127-148 (1995).

61. Owen, J. The coordination chemistry of nanocrystal surfaces. Science 347, 615 (2015).

62. Hopper, T. R. et al. Hot carrier dynamics in perovskite nanocrystal solids: role of the cold carriers, nanoconfinement, and the surface. Nano Lett. 20, 2271-2278 (2020)

63. Joshi, P. P., Maehrlein, S. F. \& Zhu, X. Dynamic screening and slow cooling of hot carriers in lead halide perovskites. Adv. Mater. 0, 1803054 (2019).

64. Frost, J. M., Whalley, L. D. \& Walsh, A. Slow cooling of hot polarons in halide perovskite solar cells. ACS Energy Lett. 2, 2647-2652 (2017).

65. Evans, T. J. S. et al. Competition between hot-electron cooling and large polaron screening in $\mathrm{CsPbBr}_{3}$ perovskite single crystals. J. Phys. Chem. C 122, 13724-13730 (2018).

66. Miyata, K. et al. Large polarons in lead halide perovskites. Sci. Adv. 3, e1701217 (2017).

\section{Acknowledgements}

This research was supported by Nanyang Technological University under its start-up grant (M4080514) and its JSPS-NTU Joint Research Project (M4082176); by the Ministry of Education under its AcRF Tier 2 grants (MOE2016-T2-1-034 and MOE2017-T2-2002); and by the National Research Foundation (NRF) Singapore under its NRF Investigatorship (NRF-NRFI-2018-04). 


\section{Author contributions}

Conceptualization, M.R., S.S.L., D.G., T.C.S.; Methodology, D.G., J.W.M.L.; Investigation, M.R., S.S.L., D.G.; Resources, M.R., Q.Z., S.R., E.Y.K.T.; Writing - Original Draft, M.R., Writing - Review \& Editing, M.R., S.S.L., D.G., T.C.S.; Visualization, M.R., S.S.L., D.G., J.W.M.L., Supervision, T.C.S.; Funding Acquisition, T.C.S.

\section{Competing interests}

The authors declare no competing interests.

\section{Additional information}

Supplementary information is available for this paper at https://doi.org/10.1038/s41467020-16463-7.

Correspondence and requests for materials should be addressed to T.C.S.

Peer review information Nature Communications thanks Chunfeng Zhang and the other, anonymous, reviewer(s) for their contribution to the peer review of this work. Peer reviewer reports are available.
Reprints and permission information is available at http://www.nature.com/reprints

Publisher's note Springer Nature remains neutral with regard to jurisdictional claims in published maps and institutional affiliations.

(c) (i) Open Access This article is licensed under a Creative Commons Attribution 4.0 International License, which permits use, sharing, adaptation, distribution and reproduction in any medium or format, as long as you give appropriate credit to the original author(s) and the source, provide a link to the Creative Commons license, and indicate if changes were made. The images or other third party material in this article are included in the article's Creative Commons license, unless indicated otherwise in a credit line to the material. If material is not included in the article's Creative Commons license and your intended use is not permitted by statutory regulation or exceeds the permitted use, you will need to obtain permission directly from the copyright holder. To view a copy of this license, visit http://creativecommons.org/ licenses/by/4.0/.

(C) The Author(s) 2020 